\title{
Brain-to-Brain Synchrony in the STEM Classroom
}

\section{Ido Davidesco*}

Department of Educational Psychology, Neag School of Education, University of Connecticut, Storrs, CT 06269

\begin{abstract}
Cognitive neuroscience research is typically conducted in controlled laboratory environments that hold very little resemblance to science, technology, engineering, and mathematics classrooms. Fortunately, recent advances in portable electroencephalography technology now allow researchers to collect brain data from groups of students in real-world classrooms. Even though this line of research is still new, there is growing evidence that students' engagement, memory retention, and social dynamics are reflected in the brain-to-brain synchrony between students and teachers (i.e., the similarity in their brain responses). In this Essay, I will provide an overview of this emerging line of research, discuss how this approach can facilitate new collaborations between neuroscientists and discipline-based education researchers, and propose directions for future research.
\end{abstract}

\section{INTRODUCTION}

With the transition from lecture-based to active-learning formats, science, technology, engineering, and mathematics (STEM) undergraduate classrooms are becoming more social (Linton et al., 2014; Eddy et al., 2015). Yet social interactions and their impact on student learning, especially at the undergraduate level, are vastly underexplored. Discipline-based education researchers typically collect data from large groups of students, but the emphasis is on how pedagogy affects individual students (Grunspan et al., 2014). There is relatively a small (but growing) number of discipline-based education research (DBER) studies that focus on social interactions. These studies suggest that social interactions can help explain shifts in students' self-efficacy (Dou et al., 2016), persistence in introductory courses (Zwolak et al., 2017), and academic performance (Theobald et al., 2017; Vargas et al., 2018).

Similarly, there is very limited neuroscience research on social interactions. In fact, the study of real-world social exchanges has been dubbed the "dark matter of social neuroscience" (Schilbach et al., 2013). The lack of studies investigating these phenomena is most likely due to the high cost and fragility of traditional cognitive neuroscience methods, limiting the vast majority of research on the human brain to studies in which one participant at a time performs a task in a highly constrained environment (e.g., inside a brain scanner). In the past few years, researchers have begun to approach the neural basis of social interactions by comparing the brain responses of multiple individuals during a variety of tasks (Hasson et al., 2012; Babiloni and Astolfi, 2014; Wheatley et al., 2019). In pioneering research, Hasson and colleagues (2004) used functional magnetic resonance imaging (fMRI) to demonstrate that the brains of different people who watch the same movie show increasingly similar activity patterns over time (a phenomenon called "brain-to-brain synchrony"). Other research has found that successful communication is associated with similar brain activity between a speaker and a listener: the more similar the listener's brain activity with that of the storyteller, the better the listener remembers a story (Stephens et al., 2010). However, participants in these studies were not tested simultaneously (i.e., there was no social interaction). The few truly interactive studies that exist demonstrate a relationship
Erin L. Dolan, Monitoring Editor

Submitted Nov 25, 2019; Revised Feb 4, 2020 Accepted Feb 5, 2020

CBE Life Sci Educ September 1, 2020 19:es8 DOI:10.1187/cbe.19-11-0258

*Address correspondence to: Ido Davidesco (ido.davidesco@uconn.edu).

(C) 2020 I. Davidesco. CBE-Life Sciences Education @ 2020 The American Society for Cell Biology. This article is distributed by The American Society for Cell Biology under license from the author(s). It is available to the public under an Attribution-Noncommercial-Share Alike 3.0 Unported Creative Commons License (http://creativecommons.org/licenses/ by-nc-sa/3.0)

"ASCB®" and "The American Society for Cell Biology $\AA^{\circledR}$ are registered trademarks of The American Society for Cell Biology. 
between social factors and brain-to-brain synchrony. For example, face-to-face dialogues have been shown to induce more brain synchrony compared with back-to-back dialogues or monologues (Jiang et al., 2012). It has also been demonstrated that conversational leaders show more synchronous brain activity with "followers" than followers do among one another (Jiang et al., 2015).

In this Essay, I will discuss how social interactions in STEM classrooms can be studied from a neuroscience perspective. Though this line of research is still at an early stage, I will argue that it holds great potential for cross-disciplinary research in biology education. I will first describe how portable technologies can be used to measure the brain activity of groups of students in classrooms and review several recent studies that used this approach. These studies suggest that students exhibit synchronized brain activity patterns, and the extent of brain synchrony reflects students' engagement, social closeness, and learning outcomes. I will then discuss what might drive brain synchrony between students in classrooms and conclude with suggestions for future research.

\section{PORTABLE BRAIN TECHNOLOGIES IN STEM EDUCATION RESEARCH}

This Essay focuses on electroencephalography (EEG), because it is currently the only brain-measuring technology that allows the recording of students' and teachers' brain activity in classrooms at just a fraction of the cost of laboratory-based neuroscience equipment. Other neuroimaging techniques, such as fMRI, positron emission tomography, and magnetoencephalography, require stationary brain scanners that cost millions of dollars. Another neuroimaging method that is gaining popularity is functional near-infrared spectroscopy (fNIRS), which uses near-infrared light to measure brain activation. Although wireless fNIRS devices are now becoming available, almost all fNIRS research to date has been conducted in laboratory settings (Cui et al., 2011).

What does EEG measure? EEG measures the brain's electrical activity from electrodes placed on the scalp (typically 32-256 electrodes). The EEG signal is thought to reflect the summation of postsynaptic potentials across thousands of neurons (Biasiucci et al., 2019; Brienza and Mecarelli, 2019). The EEG signal is primarily generated by cortical pyramidal neurons. Due to their anatomical geometry, when these neurons are activated in synchrony, their electrical signals summate and propagate to the scalp. Thus, the EEG signal varies according to the synchronized or desynchronized activity of large populations of neurons. Biasiucci et al. (2019) propose the following analogy: EEG is similar to measuring the roar of a crowd from outside a stadium. It cannot identify individual conversations, but it can detect changes in the overall activity of the crowd (for a recent primer on EEG, see Biasiucci et al., 2019).

Whereas other neuroimaging tools, such as fMRI, can detect brain activations within a range of a few millimeters, with EEG, it is mathematically very difficult to infer where in the brain the EEG signal originates. Another limitation of EEG is that the signal is often contaminated by other sorts of physiological electrical activity (e.g., ocular and other muscular activity) and environmental noise (e.g., computer screens). This is particularly a concern when using EEG in classrooms, and therefore, EEG data should be closely examined and artifacts should be identified and removed (see Dikker et al., 2017). On the other hand, EEG is very useful in determining when a certain brain response is happening, making EEG a rich source of data when it comes to disentangling different stages of information processing (Luck, 2014).

Most EEG research is still being conducted in laboratory settings, but with recent developments in low-cost, portable, wireless, and dry (i.e., gel-free) EEG technology, researchers can now conduct neuroscience investigations outside the laboratory in ecologically valid environments, such as classrooms. Biology education researchers might be especially interested in smartphone-based EEG systems that combine an off-the-shelf EEG device with a smartphone or a tablet (Debener et al., 2012; Stopczynski et al., 2014; Poulsen et al., 2017) as well as EEG systems that offer less obtrusive electrode configurations (Debener et al., 2015). However, one should note that portable EEG devices have fewer electrodes, which might restrict data analysis and the types of research questions that can be answered. Also, data acquired with portable EEG devices are more susceptible to artifacts, such as head motion and eye movement artifacts, resulting in a higher percentage of data exclusion. Nonetheless, it has been demonstrated that portable EEG devices can yield comparable data to laboratory-grade systems (at least with tasks that are known to generate robust EEG effects; Badcock et al., 2013; Ries et al., 2014; Grummett et al., 2015).

\section{BRAIN-TO-BRAIN SYNCHRONY IN CLASSROOMS}

Several recent studies have used portable EEG methods to collect brain data from groups of students in simulated and realworld classrooms. Poulsen et al. (2017) measured the brain activity of a group of 12 young adults in a classroom setting while they were presented with short video clips. The videos elicited synchronized brain activity patterns across participants compared with randomly scene-scrambled versions of the same videos. While this study helped validate the method of recording EEG in a classroom, its educational relevance is limited due to the type of materials that were used (segments of popular movies) and the fact that the participants were not an organic group of students.

In another recent study, Dikker et al. (2017) recorded EEG activity from a group of 12 students in a biology high school classroom. Students' brain activity was recorded throughout various classroom activities, such as lectures, instructional videos, and group discussions (Figure 1). The extent to which brain activity was synchronized across students was found to predict self-reported student engagement: Students who reported being more engaged exhibited higher brain synchrony with their peers. Further, brain-to-brain synchrony between pairs of students reflected how close they felt toward each other: pairs of students who demonstrated higher brain synchrony also reported higher social closeness (Dikker et al., 2017).

In another study, EEG activity was recorded not only from the students, but also from their teacher, which is a challenging task due to the sensitivity of EEG to head movement and speech-related artifacts. By instructing the teacher to be mindful of their head motion and sufficiently preprocessing the data, the authors were able to measure the brain synchrony between 


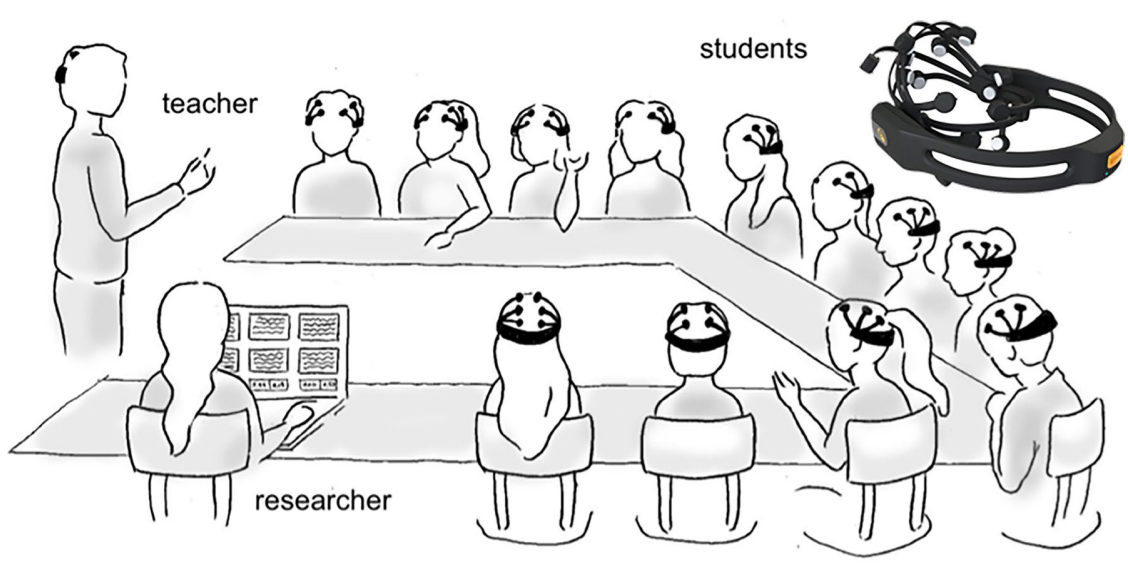

FIGURE 1. Portable EEG recordings in a classroom (from Dikker et al., 2017). Inset: An example of a portable EEG device (EMOTIV EPOC+).

\section{WHAT MIGHT GIVE RISE TO BRAIN- TO-BRAIN SYNCHRONY DURING CLASSROOM INTERACTIONS?}

While the underlying mechanisms of brain-to-brain synchrony are not well understood, Dikker et al. (2017) proposed that shared attention plays a crucial role. At the most basic level, brain-tobrain synchrony is driven by the fact that all students were exposed to the same stimulus (e.g., the instructor's voice). When confronted with an external stimulus, brain activity becomes temporally aligned to the rhythm of the input, a phenomenon called "stimulus entrainment" (Lakatos et al., 2008). In a classroom, because all students have similar sensory-motor systems designed to apprehend the world, their brains all become entrained to the instructor's voice or to

students and their teacher. Student-to-teacher brain synchrony was significantly correlated with students' self-reported engagement: Students who were more engaged showed higher brain synchrony with the teacher. Further, students who reported feeling closer to the teacher exhibited more student-to-teacher brain synchrony (Bevilacqua et al., 2019).

Taken together, these two studies suggest that the social dynamics among students and their teacher are, to some extent, reflected in their brain-to-brain synchrony. This is intriguing, because students in these studies were not prompted to think about their social relationships while EEG data were being collected. This finding is consistent with recent fMRI research, which reported that neural responses to naturalistic movies were highly similar among friends, with similarity of neural responses decreasing as social distance increased (Parkinson et al., 2018).

So far, I have focused on student engagement and social dynamics-but how does brain-to-brain synchrony relate to learning outcomes? Bevilacqua et al. (2019) did not find a significant association between brain synchrony and students' memory retention, but two other studies indicate otherwise (Cohen et al., 2018; Davidesco et al., 2019). Cohen et al. (2018) demonstrated that brain-to-brain synchrony between students who watched science-related instructional videos predicted their performance in a memory test. In another study, brain activity was concurrently measured from four non-science major students and their instructor in a simulated classroom throughout a sequence of four mini-lectures in biology and chemistry. Students' knowledge was measured a week before, immediately after, and a week following the EEG session. Both student-to-student and student-to-teacher brain synchrony significantly predicted students' memory retention a week after the lesson took place. Interestingly, moment-to-moment variations in brain synchrony throughout the lecture indicated what specific information students retained: Brain synchrony was higher for test questions for which students demonstrated learning (i.e., answered incorrectly in the pretest and correctly in the posttest) compared with test questions for which students' answers remained unchanged (Davidesco et al., 2019).
Critically, stimulus entrainment only provides a partial explanation to brain synchrony in classrooms. Our brains act as a selective filter of the external world (Enns and Lleras, 2008; Berkes et al., 2011); thus, sharing the same audiovisual input does not guarantee that we all "see" the world the same way. For example, instructors may trigger completely different responses in each of their students' brains depending on students' attention. Attention has a critical role in the learning process, as it controls the flow of incoming information by suptask-relevant information (Kanwisher and Wojciulik, 2000).

Many studies have demonstrated that attention can modulate how the brain processes information (e.g., Davidesco et al., 2013). For example, in a cocktail party-like scenario, when participants are confronted with two speakers and asked to direct attention to only one of them, brain activity in high-order auditory regions tracks only the attended speaker's voice (Mesgarani and Chang, 2012; Golumbic et al., 2013). Similarly, as students pay close attention to a lecture, their brains become entrained to the lecturer's voice, and thus their brain activity aligns with the brain activity of the instructor and other students. In contrast, as students lose interest, their brains become less entrained to the lecturer (and possibly more entrained to other stimuli), resulting in decreased similarity in neural responses to the instructor and other students (Dikker et al., 2017).

\section{BRAIN SYNCHRONY AS A REFLECTION OF INTERPERSONAL COORDINATION}

During social interactions, not only do people's brains become synchronized, but also their behaviors become aligned (Cornejo et al., 2017). For example, during conversations, people tend to imitate each other's choices of speech sounds, grammatical forms, and words, a process known as "interactive alignment" (Garrod and Pickering, 2004). Interpersonal coordination also occurs nonverbally: Interlocutors tend to synchronize their facial expressions, manual gestures, and noncommunicative postures. This typically happens spontaneously, unconsciously, and rapidly (within a few seconds after the onset of an interaction; Louwerse et al., 2012). any other shared stimuli, thus syncing with one another. pressing irrelevant information while enhancing sensitivity to 
Even though teaching and learning are highly social processes, with students and teachers interacting constantly with one another, there is very little research on interpersonal coordination in classrooms. In one of the few studies that addressed this issue, Bernieri (1988) measured the movement synchrony and behavioral matching in video recordings of high school students. The students were videotaped in pairs as they attempted to teach each other a set of imaginary words. Ratings of movement synchrony and behavioral matching were higher in genuine interaction video clips compared with control video clips. Further, the degree of movement and behavioral synchrony were positively correlated with rapport ratings between students. In another more recent study, bilingual undergraduate and graduate students engaged in teaching-like tasks, where one person was required to transmit information that was unknown to their partner in order to achieve a shared goal. An independent group of native English speakers rated the degree of alignment between interlocutors. Interactive alignment in both linguistic (e.g., word stress placement in multisyllabic words) and nonverbal behaviors was found to be significantly higher at the end of the conversation compared with the beginning, suggesting that interpersonal coordination increases over time (Trofimovich and Kennedy, 2014; Trofimovich et al., 2014).

Interpersonal coordination relies on our ability to anticipate both our own linguistic and behavioral actions and those of others (Ramnani and Miall, 2004; Sebanz et al., 2006; Konvalinka et al., 2010; Sänger et al., 2011; Romero et al., 2012; Pickering and Garrod, 2013). For example, using fMRI, Stephens et al. (2010) reported that neural responses in the frontal cortex of the listener's brain preceded the responses in the speaker's brain. These anticipatory responses suggest that listeners are actively generating predictions of what the speaker is about to say. Interestingly, the extent of brain areas where the listeners' activity preceded the speaker's activity was found to be the best predictor of listeners' comprehension of the story to which they listened (Stephens et al., 2010). Similarly, Davidesco et al. (2019) demonstrated that in frontal and central EEG locations, student-to-teacher brain synchrony best predicted learning outcomes when the students' brain responses preceded the teachers' brain responses. However, the role of prior knowledge in this process is not clear. It is possible that students who knew more about the topic before the lecture were better able to generate predictions about what the instructor was about to say, and that allowed them to learn more efficiently.

\section{CONCLUSIONS AND NEXT STEPS}

Social interactions in STEM classrooms as well as how they are reflected in students' brain activity seem to be promising directions for interdisciplinary research in biology education. Social interactions are often overlooked in DBER, despite having a direct impact on students' academic performance (Linton et al., 2014; Theobald et al., 2017; Vargas et al., 2018). Similarly, social interactions are understudied in human neuroscience, where most research is conducted on individual participants in controlled laboratory environments.

This emerging line of research provides an opportunity for cross-disciplinary collaborations between discipline-based education researchers and cognitive and social neuroscientists (Davidesco and Milne, 2019; Mestre et al., 2018). The connec- tion between neuroscience and education has been described in the past as "a bridge too far" (Bruer, 1997). Since then, there has been growing interest and debate regarding the relevance of cognitive neuroscience to education research and practice (Ansari and Coch, 2006; Goswami, 2006; Sigman et al., 2014; Bowers, 2016). Bridging neuroscience and education is challenging, because these disciplines have very different goals and research traditions. Neuroscientists typically adopt a reductionist approach and study cognitive functions in isolation. Educational researchers, on the other hand, focus on the learner as a whole and how the learner is embedded in a context, such as a classroom. A common concern is that traditional cognitive neuroscience methods (e.g., fMRI) are conducted in artificial laboratory environments and therefore cannot provide useful data to understand real-world learning. From a more pragmatic standpoint, the concern is that neuroscience methods are just too expensive to apply to educational research (Varma et al., 2008).

This Essay highlighted recent developments in portable and wearable brain technologies, such as portable EEG, which now allow researchers to use neuroscience methods in classroom-based research at just a fraction of the cost (Dikker et al., 2017; Bevilacqua et al., 2019). These methods can complement other measures used in DBER, such as achievement tests, self-reports, and think-aloud interviews, and can deepen our understanding of the learning process in several important ways. First, neuroscience methods provide continuous data without interfering with naturally occurring learning activities. Using these methods, we can better explore the cognitive processing that is taking place during learning, rather than just before and after learning (Mayer, 2017). Further, these methods can potentially measure implicit processes that learners are unaware of or unable to report accurately, such as lapses of attention (Dahlstrom-Hakki et al., 2019). Finally, neuroscientific data can be used to explore individual differences that mediate learning and predict how students would benefit from different pedagogies (Gabrieli, 2016; Mayer, 2017).

Brain-to-brain synchrony is an example of what can be measured in neuroscience research in STEM classrooms, but this phenomenon is not unique to STEM. In fact, it is not unique to classrooms at all, but likely occurs in any type of social interaction (Hasson et al., 2012). But in the case of classroom learning, brain-to-brain synchrony can be an informative measure of student engagement, social dynamics, and learning outcomes (Dikker et al., 2017; Cohen et al., 2018; Bevilacqua et al., 2019; Davidesco et al., 2019).

However, there are several inherent limitations associated with classroom-based EEG research. First, EEG is a correlative method, where neural activity is associated with behavioral measures (e.g., test scores). Thus, EEG cannot assess causal relationships. Second, the low spatial resolution of EEG limits the information available to researchers. Questions about what specific brain regions are involved in classroom learning cannot currently be addressed with EEG. Third, EEG recordings are sensitive to muscle-related artifacts caused by head motion, eye movements, and speech. For this reason, classroom-based EEG research to date has focused primarily on passive forms of learning, such as listening to lectures and watching instructional videos, where student movement and speech are limited (Dikker et al., 2017; Bevilacqua et al., 2019). However, now 
that this method has been successfully deployed in classrooms, future research can start exploring more interactive forms of learning, such as small-group work.

Recent calls to enhance active learning in STEM education have emphasized group work based on the understanding that collaborative groups can enhance learning, improve students' attitudes toward science, and reinforce their social identity as scientists (Springer et al., 1999; Tanner et al., 2003; National Research Council, 2012; President's Council of Advisors on Science and Technology, 2012). However, groups can be dysfunctional, and we do not know enough about how to best use group learning in STEM education, especially at the college level (Theobald et al., 2017). Future cross-disciplinary research teams can collect both EEG and audio-video data from small groups of students during collaborative learning tasks. Whereas previous work has typically measured brain-to-brain synchrony throughout the entire duration of an activity, the high temporal resolution of EEG allows researchers to examine how brain synchrony (as well as other EEG measures) unfolds over time. For example, researchers can identify moments of high and low synchrony in the EEG data and examine the corresponding audio and video recordings to identify any recurring patterns. This research might generate new insights into what makes group learning effective.

In summary, I believe that neuroscience research in classrooms provides an exciting opportunity for both discipline-based education researchers and cognitive and social neuroscientists. From a DBER perspective, this research will hopefully yield a deeper, more mechanistic understanding of STEM learning by illuminating what is happening in students' brains during the learning process and what factors mediate learning. From a neuroscience perspective, this research provides a unique opportunity to study the brain mechanisms that support learning in real-world classroom environments.

\section{ACKNOWLEDGMENTS}

This work has been supported by NSF grant no. 1661016. The illustration in Figure 1 is reprinted from Current Biology, 27(9), Dikker et al., "Brain-to-brain synchrony tracks real-world dynamic group interactions in the classroom," pages 1375 1380, Copyright (2017), with permission from Elsevier.

\section{REFERENCES}

Ansari, D., \& Coch, D. (2006). Bridges over troubled waters: Education and cognitive neuroscience. Trends in Cognitive Sciences, 10(4), 146-151.

Babiloni, F., \& Astolfi, L. (2014). 2014). Social neuroscience and hyperscanning techniques: Past, present and future. Neuroscience \& Biobehavioral Reviews, 44, 76-93

Badcock, N. A., Mousikou, P., Mahajan, Y., De Lissa, P., Thie, J., \& McArthur, G. (2013). Validation of the Emotiv EPOC $®$ EEG gaming system for measuring research quality auditory ERPs. PeerJ, 1, e38.

Berkes, P., Orbán, G., Lengyel, M., \& Fiser, J. (2011). Spontaneous cortical activity reveals hallmarks of an optimal internal model of the environment. Science, 331(6013), 83-87.

Bernieri, F. J. (1988). Coordinated movement and rapport in teacher-student interactions. Journal of Nonverbal Behavior, 12(2), 120-138.

Bevilacqua, D., Davidesco, I., Wan, L., Chaloner, K., Rowland, J., Ding, M., ... \& Dikker, S. (2019). Brain-to-brain synchrony and learning outcomes vary by student-teacher dynamics: Evidence from a real-world classroom electroencephalography study. Journal of Cognitive Neuroscience, 31(3), 401-411.

Biasiucci, A., Franceschiello, B., \& Murray, M. M. (2019). Electroencephalography. Current Biology, 29(3), R80-R85.
Bowers, J. S. (2016). The practical and principled problems with educational neuroscience. Psychological Review, 123(5), 600-612.

Brienza, M., \& Mecarelli, O. (2019) Neurophysiological basis of EEG. In Mecarelli, O. (Ed.), Clinical electroencephalography. Cham, Switzerland: Springer.

Bruer, J. T. (1997). Education and the brain: A bridge too far. Educational Researcher, 26(8), 4-16.

Cohen, S. S., Madsen, J., Touchan, G., Robles, D., Lima, S. F. A., Henin, S., \& Parra, L. C. (2018). Neural engagement with online educational videos predicts learning performance for individual students. Neurobiology of Learning and Memory, 155, 60-64.

Cornejo, C., Cuadros, Z., Morales, R., \& Paredes, J. (2017). Interpersonal coordination: Methods, achievements, and challenges. Frontiers in Psychology, 8, 1685

Cui, X., Bray, S., Bryant, D. M., Glover, G. H., \& Reiss, A. L. (2011). A quantitative comparison of NIRS and fMRI across multiple cognitive tasks. Neuroimage, 54(4), 2808-2821.

Dahlstrom-Hakki, I., Asbell-Clarke, J., \& Rowe, E. (2019). Showing is knowing The potential and challenges of using neurocognitive measures of implicit learning in the classroom. Mind, Brain, and Education, 13(1), 30-40.

Davidesco, I., Harel, M., Ramot, M., Kramer, U., Kipervasser, S., Andelman, F. ... \& Malach, R. (2013). Spatial and object-based attention modulates broadband high-frequency responses across the human visual cortical hierarchy. Journal of Neuroscience, 33(3), 1228-1240.

Davidesco, I., Laurent, E., Valk, H., West, T., Dikker, S., Milne, C., \& Poeppel, D. (2019). Brain-to-brain synchrony between students and teachers predicts learning outcomes. Retrieved from bioRxiv 644047. doi: $10.1101 / 644047$

Davidesco, I., \& Milne, C. (2019). Implementing cognitive science and discipline-based education research in the undergraduate science classroom. CBE-Life Sciences Education, 18(3), es4.

Debener, S., Emkes, R., De Vos, M., \& Bleichner, M. (2015). Unobtrusive ambulatory EEG using a smartphone and flexible printed electrodes around the ear. Scientific Reports, 5, 16743

Debener, S., Minow, F., Emkes, R., Gandras, K., \& De Vos, M. (2012). How about taking a low-cost, small, and wireless EEG for a walk? Psychophysiology, 49(11), 1617-1621.

Dikker, S., Wan, L., Davidesco, I., Kaggen, L., Oostrik, M., McClintock, J., ... \& Poeppel, D. (2017). Brain-to-brain synchrony tracks real-world dynamic group interactions in the classroom. Current Biology, 27(9), 1375-1380.

Dou, R., Brewe, E., Zwolak, J. P., Potvin, G., Williams, E. A., \& Kramer, L. H. (2016). Beyond performance metrics: Examining a decrease in students physics self-efficacy through a social networks lens. Physical Review Physics Education Research, 12(2), 020124.

Eddy, S. L., Brownell, S. E., Thummaphan, P., Lan, M.-C., \& Wenderoth, M. P. (2015). Caution, student experience may vary: Social identities impact a student's experience in peer discussions. CBE-Life Sciences Education, 14(4), ar45.

Enns, J. T., \& Lleras, A. (2008). What's next? New evidence for prediction in human vision. Trends in Cognitive Sciences, 12(9), 327-333.

Gabrieli, J. D. (2016). The promise of educational neuroscience: Comment on Bowers (2016). Psychological Review, 123(5), 613-619.

Garrod, S., \& Pickering, M. J. (2004). Why is conversation so easy? Trends in Cognitive Sciences, 8(1), 8-11.

Golumbic, E. M. Z., Ding, N., Bickel, S., Lakatos, P., Schevon, C. A., McKhann, G. M., ... \& Simon, J. Z. (2013). Mechanisms underlying selective neuronal tracking of attended speech at a "cocktail party." Neuron, 77(5), 980-991.

Goswami, U. (2006). Neuroscience and education: From research to practice? Nature Reviews Neuroscience, 7(5), 406-413.

Grummett, T., Leibbrandt, R., Lewis, T., DeLosAngeles, D., Powers, D. Willoughby, J., ... \& Fitzgibbon, S. (2015). Measurement of neural signals from inexpensive, wireless and dry EEG systems. Physiological Measurement, 36(7), 1469-1484.

Grunspan, D. Z., Wiggins, B. L., \& Goodreau, S. M. (2014). Understanding classrooms through social network analysis: A primer for social network analysis in education research. CBE-Life Sciences Education, 13(2), 167-178.

Hasson, U., Ghazanfar, A., Galantucci, B., Garrod, S., \& Keysers, C. (2012) Brain-to-brain coupling: A mechanism for creating and sharing a social world. Trends in Cognitive Sciences, 16(2), 114-121. 
Hasson, U., Nir, Y., Levy, I., Fuhrmann, G., \& Malach, R. (2004). Intersubject synchronization of cortical activity during natural vision. Science, 303(5664), 1634-1640.

Jiang, J., Chen, C., Dai, B., Shi, G., Ding, G., Liu, L., \& Lu, C. (2015). Leader emergence through interpersonal neural synchronization. Proceedings of the National Academy of Sciences USA, 112(14), 4274-4279.

Jiang, J., Dai, B., Peng, D., Zhu, C., Liu, L., \& Lu, C. (2012). Neural synchronization during face-to-face communication. Journal of Neuroscience, 32(45), 16064-16069.

Kanwisher, N., \& Wojciulik, E. (2000). Visual attention: Insights from brain imaging. Nature Reviews Neuroscience, 1(2), 91-100.

Konvalinka, I., Vuust, P., Roepstorff, A., \& Frith, C. D. (2010). Follow you, follow me: Continuous mutual prediction and adaptation in joint tapping. Quarterly Journal of Experimental Psychology, 63(11), 2220-2230.

Lakatos, P., Karmos, G., Mehta, A. D., Ulbert, I., \& Schroeder, C. E. (2008). Entrainment of neuronal oscillations as a mechanism of attentional selection. Science, 320(5872), 110-113.

Linton, D. L., Farmer, J. K., \& Peterson, E. (2014). Is peer interaction necessary for optimal active learning? CBE-Life Sciences Education, 13(2), 243252.

Louwerse, M. M., Dale, R., Bard, E. G., \& Jeuniaux, P. (2012). Behavior matching in multimodal communication is synchronized. Cognitive Science, 36(8), 1404-1426.

Luck, S. J. (2014). An introduction to the event-related potential technique. Cambridge, MA: MIT Press.

Mayer, R. E. (2017). How can brain research inform academic learning and instruction? Educational Psychology Review, 29(4), 835-846.

Mesgarani, N., \& Chang, E. F. (2012). Selective cortical representation of attended speaker in multi-talker speech perception. Nature, 485(7397), $233-236$.

Mestre, J. P., Cheville, A., \& Herman, G. L. (2018). Promoting DBER-cognitive psychology collaborations in STEM education. Journal of Engineering Education, 107(1), 5-10.

National Research Council (2012). Discipline-based education research. Washington, DC: National Academies Press.

Parkinson, C., Kleinbaum, A. M., \& Wheatley, T. (2018). Similar neural responses predict friendship. Nature Communications, 9(1), 332.

Pickering, M. J., \& Garrod, S. (2013). An integrated theory of language production and comprehension. Behavioral and Brain Sciences, 36(04), 329-347.

Poulsen, A. T., Kamronn, S., Dmochowski, J., Parra, L. C., \& Hansen, L. K. (2017). EEG in the classroom: Synchronised neural recordings during video presentation. Scientific Reports, 7, 43916.

President's Council of Advisors on Science and Technology. (2012). Engage to excel: Producing one million additional college graduates with degrees in science, technology, engineering, and mathematics. Washington, DC: U.S. Government Office of Science and Technology.

Ramnani, N., \& Miall, R. C. (2004). A system in the human brain for predicting the actions of others. Nature Neuroscience, 7(1), 85-90.

Ries, A. J., Touryan, J., Vettel, J., McDowell, K., \& Hairston, W. D. (2014). A comparison of electroencephalography signals acquired from conventional and mobile systems. Journal of Neuroscience and Neuroengineering, 3(1), 10-20.

Romero, V., Coey, C., Schmidt, R. C., \& Richardson, M. J. (2012). Movement coordination or movement interference: Visual tracking and spontaneous coordination modulate rhythmic movement interference. PLoS ONE, 7(9), e44761.

Sänger, J., Lindenberger, U., \& Müller, V. (2011). Interactive brains, social minds. Communicative \& Integrative Biology, 4(6), 655-663.

Schilbach, L., Timmermans, B., Reddy, V., Costall, A., Bente, G., Schlicht, T., \& Vogeley, K. (2013). Toward a second-person neuroscience. Behavioral and Brain Sciences, 36(4), 393-414

Sebanz, N., Bekkering, H., \& Knoblich, G. (2006). Joint action: Bodies and minds moving together. Trends in Cognitive Sciences, 10(2), 70-76.

Sigman, M., Peña, M., Goldin, A. P., \& Ribeiro, S. (2014). Neuroscience and education: Prime time to build the bridge. Nature Neuroscience, 17(4), 497-502.

Springer, L., Stanne, M. E., \& Donovan, S. S. (1999). Effects of small-group learning on undergraduates in science, mathematics, engineering, and technology: A meta-analysis. Review of Educational Research, 69(1), 21-51.

Stephens, G. J., Silbert, L. J., \& Hasson, U. (2010). Speaker-listener neural coupling underlies successful communication. Proceedings of the $\mathrm{Na}$ tional Academy of Sciences USA, 107(32), 14425

Stopczynski, A., Stahlhut, C., Larsen, J. E., Petersen, M. K., \& Hansen, L. K. (2014). The smartphone brain scanner: A portable real-time neuroimaging system. PLOS ONE, 9(2), e86733.

Tanner, K., Chatman, L. S., \& Allen, D. (2003). Approaches to cell biology teaching: Cooperative learning in the science classroom-beyond students working in groups. Cell Biology Education, 2(1), 1-5.

Theobald, E. J., Eddy, S. L., Grunspan, D. Z., Wiggins, B. L., \& Crowe, A. J. (2017). Student perception of group dynamics predicts individual performance: Comfort and equity matter. PLOS ONE, 12(7), e0181336.

Trofimovich, P., \& Kennedy, S. (2014). Interactive alignment between bilingual interlocutors: Evidence from two information-exchange tasks. Bilingualism: Language and Cognition, 17(4), 822-836.

Trofimovich, P., McDonough, K., \& Foote, J. A. (2014). Interactive alignment of multisyllabic stress patterns in a second language classroom. TESOL Quarterly, 48(4), 815-832

Vargas, D. L., Bridgeman, A. M., Schmidt, D. R., Kohl, P. B., Wilcox, B. R., \& Carr L. D. (2018). Correlation between student collaboration network centrality and academic performance. Physical Review Physics Education Research, 14(2), 020112

Varma, S., McCandliss, B. D., \& Schwartz, D. L. (2008). Scientific and pragmatic challenges for bridging education and neuroscience. Educational Researcher, 37(3), 140-152.

Wheatley, T., Boncz, A., Toni, I., \& Stolk, A. (2019). Beyond the isolated brain The promise and challenge of interacting minds. Neuron, 103(2), 186188

Zwolak, J. P., Dou, R., Williams, E. A., \& Brewe, E. (2017). Students' network integration as a predictor of persistence in introductory physics courses. Physical Review Physics Education Research, 13(1), 010113 